\title{
Photorecombination and Photoionization Experiments at Heavy-Ion Storage-Rings and Synchrotron-Light Sources
}

\author{
Stefan Schippers \\ Institut für Kernphysik, Justus-Liebig-Universität Giessen, \\ Leihgesterner Weg 217, 35392 Giessen, Germany
}

\begin{abstract}
Recent experimental work on the photorecombination and the photoionization of astrophysically relevant atomic ions employing the mergedbeams technique at heavy-ion storage-rings and synchrotron-light sources, respectively, is summarized. The resulting absolute photoionization cross sections and recombination rate coefficients benchmark corresponding theoretical calculations and are needed for the accurate determination of ionization equilibria in astrophysical plasmas.
\end{abstract}

\section{Introduction}

Photoionization (PI) and Photorecombination (PR) are basic atomic processes that govern the charge state balance in any plasma. Therefore, the accurate knowledge of these processes is a prerequisite for any plasma modeling and, hence, for any meaningful interpretation of many astrophysical observations. To date, most PI and PR cross sections used in plasma modeling stem from theoretical calculations. Their accuracy is often difficult to assess and, therefore, experimental PI and PR cross sections and rate coefficients are vitally needed as benchmarks and guidelines for the development of the theoretical methods. Moreover, in the near future, experiments will probably be the only reliable source for PR and PI cross sections of complex ions such as ions with an open M-shell (Schippers et al. 2002a; 2003a).

The measurement of PR and PI cross sections of atomic (and molecular) ions is experimentally challenging because the ion densities that can be experimentally prepared are very low - some $10^{6} \mathrm{~cm}^{-3}$ (to be compared with $\approx 10^{13} \mathrm{~cm}^{-3}$ for gaseous and $\approx 10^{22} \mathrm{~cm}^{-3}$ for solid targets). Consequently, signal rates from electron-ion and photon-ion collision experiments are comparatively weak. In order to make up for the low ion density in such experiments an - arrangement is chosen where the colliding particle beams are merged collinearly over a distance of the order of $1 \mathrm{~m}$. The merged-beams arrangement (Phaneuf et al. 1999) provides a relatively large interaction volume, and the directionality of the ion beam facilitates the effective collection of the PI or PR reaction products, i. e., of ions that have changed their charge state due to either ionization or recombination. For the measurement of absolute PR and PI cross sections the merged-beams method was implemented at heavy-ion storage rings (Müller \& Wolf 1997; Müller \& Schippers 2001) and at synchrotron-light sources (West 2001; Covington et al. 2002), respectively. 


\section{Photorecombination of atomic ions}

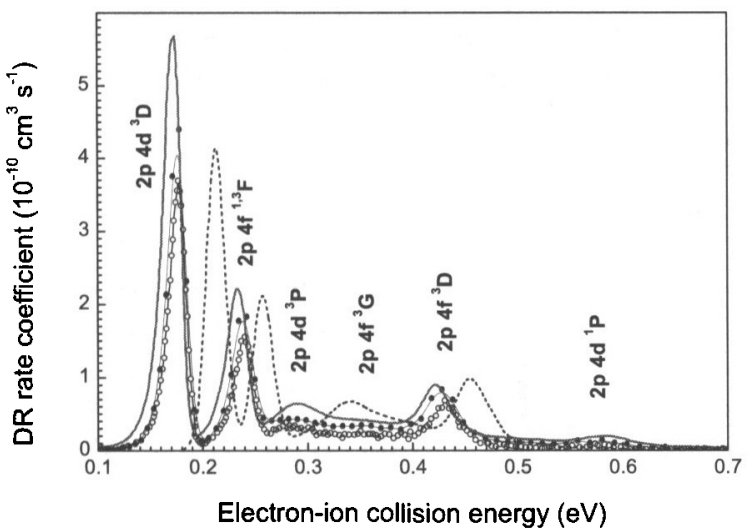

Figure 1. Dielectronic recombination (DR) of $\mathrm{C}^{3+}\left(1 s^{2} 2 s\right)$-ions at low energies: experiment (full symbols: Schippers et al. 2001, open symbols: Mannervik et al. 1998), many body perturbation theory (full line: Mannervik et al. 1998) and Breit-Pauli R-Matrix theory (dashed line: Pradhan et al. 2001).

The basic procedure for producing plasma rate coefficients from PR measurements at storage rings was outlined by Müller (1999). Recent examples are the PR rate coefficients of the lithium-like ions CIV (Schippers et al. 2001), O VI (Böhm et al. 2003) and Nixxvi (Schippers et al. 2000). Figure 1 shows a comparison between measured and calculated C IV dielectronic recombination (DR) rate coefficients at low electron-ion collision energies. The two experimental results, which were obtained a two different storage-rings, agree with one another to within the $15 \%$ experimental error for the absolute cross section. This exemplifies the reliability of recombination measurements at storage rings. Mannervik et al. (1998) also calculated the CIV DR rate coefficient using relativistic many-body perturbation theory (RMBPT, Tokman et al. 2002). They pointed out that even for a light ion such as $\mathrm{C}$ IV, a relativistic treatment of the recombination process is necessary to reproduce the experimentally observed $2 p 4 l \mathrm{DR}$ resonance structure. Presently, RMBPT is only applicable to relatively simple systems and it is therefore instructive to see in how far more standard "production" codes are able to reproduce low-energy resonance positions and strength. For C IV the Breit-Pauli R-Matrix results of Pradhan et al. (2001) differ by less than $20 \mathrm{meV}$ from the measured resonance positions (figure 1). It should be kept in mind, however, that there are pathological systems, e. g. $\mathrm{Mg} \mathrm{IX}$, where a mere $50 \mathrm{meV}$ uncertainty of low-energy resonance positions can translate into a nearly one-order-of-magnitude uncertainty on the plasma rate-coefficient scale in a temperature range where the ion is expected to form in photoionized gases (Schippers et al. 2004). Such findings strongly emphasize the need for experimental benchmarks, especially for low-temperature PR rate coefficients.

Photorecombination measurements responding to astrophysical data needs were carried out for L-shell iron ions at the Heidelberg storage-ring TSR by Savin et al. (1997; 1999; 2002a; 2002b; 2003) who already published DR rate 
coefficients for the ions FeXVIII through FexXII. In a series of measurements with lithium-like ions, the influence of external electromagnetic fields on DR cross sections was thoroughly investigated by Bartsch et al. $(1997 ; 1999 ; 2000)$, Böhm et al. (2001; 2002) and Schippers et al. (2000). This work is also of astrophysical interest. It was recently summarized by Müller \& Schippers (2003).

\section{Photoionization of atomic ions}

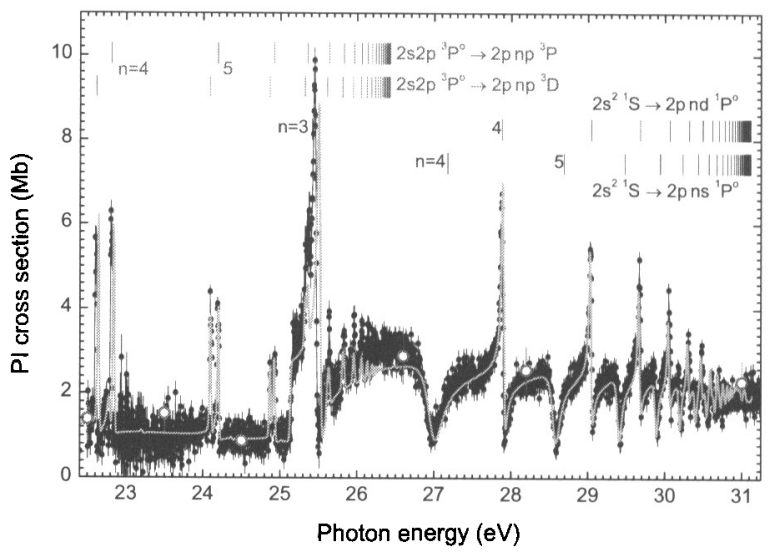

Figure 2. Photoionization of $\mathrm{B}^{+}$-ions: experiment and Breit-Pauli RMatrix theory (Schippers et al. 2003b). From the comparison between experiment and theory, it is concluded that $29 \%$ of the ions in the experiment were in the metastable state.

More recently, experiments aiming at measuring absolute PI cross sections of atomic ions and employing merged photon-ion beams were set up at 3rd generation synchrotron light sources. Measurements of astrophysical relevance were carried out, e.g., for singly charged boron (figure 2, Schippers et al. 2003b), carbon (Kjeldsen et al. 2001), nitrogen (Kjeldsen et al. 2002a), oxygen (Covington et al. 2001; Kjeldsen et al. 2002a; Aguilar et al. 2003a), neon (Covington et al. 2002), magnesium (Kjeldsen et al. 2000; Aguilar et al. 2003b) and iron (Kjeldsen et al. 2002b) as well as for the multiply charged ions C III (Müller et al. 2002), Ne IV (Aguilar 2003c), and Al III (Aguilar et al. 2003b).

Finally, it should be mentioned that the possibility to relate the time-inverse processes of PI and PR via the principle of detailed balance can be exploited for consistency checks between PI and PR measurements (Müller et al. 2002) and to obtain more comprehensive results than from only one experiment alone (Schippers et al. 2002b, 2003a).

Acknowledgments. The experimental work summarized in this article was carried out in close collaborations with S. Böhm, C. Brandau, S. Kieslich, W. Shi, and A. Müller (Gießen), D. W. Savin (New York), G. Gwinner, M. Schnell, D. Schwalm, and A. Wolf (Germany), H. Danared, N. Eklöw, and R. Schuch (Stockholm), A. Aguilar, A. M. Covington, E. D. Emmons, M. F. Gharaibeh, and R. A. Phaneuf (Reno). 


\section{References}

Aguilar, A., et al. 2003a, ApJS, 146, 467

Aguilar, A., et al. 2003b, Phys.Rev.A, 67, 012701

Aguilar, A. 2003c, PhD Thesis, (Reno: University of Nevada).

Bartsch, T., et al. 1997, Phys.Rev.Lett, 79, 2233

Bartsch, T., et al. 1999, Phys.Rev.Lett, 82, 3779

Bartsch, T., et al. 2000, J.Phys.B, 33, L453

Böhm, S., et al. 2001, Phys.Rev.A, 64, 032707

Böhm, S., et al. 2002, Phys.Rev.A, 65, 052728

Böhm, S., et al. 2003, A\&A, 405, 1157

Covington, A. M., et al. 2001, Phys.Rev.Lett, 87, 243002

Covington, A. M., et al. 2002, Phys.Rev.A, 66, 062710

Kjeldsen, H., et al. 2000, J.Phys.B, 33, 1403

Kjeldsen, H., et al. 2001, ApJS, 135, 285

Kjeldsen, H., et al. 2002a, ApJS, 138, 219

Kjeldsen, H., et al. 2002b, J.Phys.B, 35, 3655

Mannervik, S., et al. 1998, Phys.Rev.Lett, 81, 313

Müller, A., \& Wolf, A. 1997, in Accelerator-based atomic physics techniques and applications, ed. J. C. Austin \& S. M. Shafroth (Woodbury: AIP) 147

Müller, A. 1999, Int.J.Mass.Spectrom., 192, 9

Müller, A., et al. 2002, J.Phys.B, 35, L137

Müller A. \& Schippers, S. 2001, in ASP Conf. Ser. Vol. 247, Spectroscopic Challenges of Photoionized Plasmas, ed. G. Ferland \& D. W. Savin, 53

Müller, A., \& Schippers, S. 2003, in The Physics of Multiply and Highly Charged Ions, Volume 1, ed. F. J. Currell (Dordrecht: Kluwer) 269.

Phaneuf, R. A., et al. 1999, Rep.Prog.Phys, 62, 1143

Pradhan, A. K., et al. 2001, Phys.Rev.Lett, 87, 183201

Savin, D. W., et al. 1997, ApJ, 489, L115

Savin, D. W., et al. 1999, ApJS, 123, 687

Savin, D. W, et al. 2002a, ApJS, 138, 337

Savin, D. W., et al. 2002b, ApJ, 576, 1098

Savin, D. W., et al. 2003, ApJS, 147, 421

Schippers, S., et al. 2000, Phys.Rev.A, 62, 022708

Schippers, S., et al. 2001, ApJ, 555, 1027

Schippers, S., et al. 2002a, Phys.Rev.A, 66, 042723

Schippers, S., et al. 2002b, Phys.Rev.Lett, 89, 193002

Schippers, S., et al. 2003a, Phys.Rev.A, 67, 032702

Schippers, S., et al. 2003b, J.Phys.B, 36, 3371

Schippers, S., et al. 2004, A\&A, 421, 1185

Tokman, M., et al. 2002, Phys.Rev.A, 66, 012703

West, J. 2001, J.Phys.B, 34, R45 